\title{
ON A QUESTION OF DIKRANJAN AND ZAVA
}

\author{
IGOR PROTASOV
}

\begin{abstract}
Let $G$ be a non-discrete countable metrizable abelian topological group endowed with the coarse structure $\mathcal{C}$ generated by compact subsets of $G$. We prove that $\operatorname{asdim}(G, \mathcal{C})=\infty$. For an infinite cyclic subgroup $G$ of the circle, this answers a question of Dikranjan and Zava [3].
\end{abstract}

MSC: 22A15, 54E35.

Keywords: coarse structure, group ideal, asymptotic dimension.

\section{INTRODUCTION}

Let $X$ be a set. A family $\mathcal{E}$ of subsets of $X \times X$ is called a coarse structure if

- $\triangle_{X} \in \mathcal{E}, \triangle_{X}=\{(x, x): x \in X\}$;

- if $E, E^{\prime} \in \mathcal{E}$ then $E \circ E^{\prime} \in \mathcal{E}$ and $E^{-1} \in \mathcal{E}$, where $E \circ E^{\prime}=\{(x, y)$ : $\left.\exists z\left((x, z) \in E, \quad(z, y) \in E^{\prime}\right)\right\}, E^{-1}=\{(y, x):(x, y) \in E\}$;

- if $E, E^{\prime} \in \mathcal{E}$ and $H \subseteq E$ then $E \cup E^{\prime} \in E$ and $H \in \mathcal{E}$.

A subset $\mathcal{E}^{\prime} \subseteq \mathcal{E}$ is called a base for $\mathcal{E}$ if, for every $E \in \mathcal{E}$, there exists $E^{\prime} \in \mathcal{E}^{\prime}$ such that $E \subseteq E^{\prime}$. For $x \in X, A \subseteq X$ and $E \in \mathcal{E}$, we denote $E[x]=\{y \in X$ : $(x, y) \in E\}, E[A]=\cup_{a \in A} E[a]$ and say that $E[x]$ and $E[A]$ are balls of radius $E$ around $x$ and $A$.

The pair $(X, \mathcal{E})$ is called a coarse space [12]. We note that coarse spaces defined in terms of balls were introduced under the name balleans in [8] independently and simultaneously with [12], for the history see [2].

Each subset $Y \subseteq X$ defines the subspace $\left(Y, \mathcal{E}_{Y}\right)$, where $\mathcal{E}_{Y}$ is the restriction of $\mathcal{E}$ to $Y \times Y$. A subset $Y$ is called bounded if $Y \subseteq E[x]$ for some $x \in X$ and $E \in \mathcal{E}$.

A family $\mathcal{F}$ of subsets of $X$ is called $E$-bounded (E-disjoint) if, for each $A \in \mathcal{F}$, there exists $x \in X$ such that $A \subseteq E[x](E[A] \cap B=\emptyset$ for all distinct $A, B \in \mathcal{F})$.

By the definition $[12$, Chapter 9], $\operatorname{asdim}(X, \mathcal{E}) \leq n$ if, for each $E \in \mathcal{E}$, there exist $F \in \mathcal{E}$ and $F$-bounded covering $\mathcal{M}$ of $X$ which can be partitioned $\mathcal{M}=$ $\mathcal{M}_{0} \cup \ldots \cup \mathcal{M}_{n}$ so that each family $\mathcal{M}_{i}$ is $E$-disjoint. If there exists the minimal $n$ with this property then $\operatorname{asdim}(X, \mathcal{E})=n$, otherwise $\operatorname{asdim}(X, \mathcal{E})=\infty$.

Given two coarse spaces $(X, \mathcal{E}),\left(X^{\prime}, \mathcal{E}^{\prime}\right)$, a mapping $f: X \longrightarrow X^{\prime}$ is called macro-uniform (or bornologous [12]) if, for each $E \in \mathcal{E}$, there exists $E^{\prime} \in \mathcal{E}^{\prime}$ such 
that $f(E[x]) \subseteq E^{\prime}[f(x)]$ for each $x \in X$. If $f$ is a bijection such that $f$ and $f^{-1}$ are macro-uniform then $f$ is called an asymorphism.

Now let $G$ be a group. A family $\mathcal{I}$ of subsets of $G$ is called a group ideal [9], [1] if $G$ contains the family $[G]^{<\omega}$ of all finite subsets of $G$ and $A, B \in \mathcal{I}, \quad C \subseteq A$ imply $A B^{-1} \in \mathcal{I}, C \in \mathcal{I}$. Every group ideal $\mathcal{I}$ defines a coarse structure on $G$ with the base $\left\{\{(x, y): x \in A y\} \cup \triangle_{G}: A \in \mathcal{I}\right\}$. We denote $G$ endowed with this coarse structure by $(G, \mathcal{I})$.

If $G$ is discrete then the coarse space $\left(G,[G]^{<\omega}\right)$ is the main subject of Geometric Group Theory, see [4]. For coarse structures on $G$ defined by the ideal $[G]^{<\kappa}$, where $\kappa$ is a cardinal, see [10].

Every topological group $G$ can be endowed with a coarse structure defined by the ideal of all totally bounded subsets of $G$. These coarse structures were introduced and studied in [5]. For asymptotic dimensions of locally compact abelian groups endowed with coarse structures defined by ideals of precompact subsets see [6]. For the coarse structure on a topological group $G$ defined by the group ideal generated by converging sequences, see [7].

For a topological group $G$, we denote by $\mathcal{C}$ the group ideal of precompact subsets of $G$ ( $A$ is precompact if $c l A$ is compact). In [3, Problem 5.1] Dikranjan and Zava observed that $\operatorname{asdim}(G, \mathcal{C})>0$ for an infinite cyclic subgroup of the circle and asked about the value of $\operatorname{asdim}(G, \mathcal{C})$.

\section{Results}

We denote by $\Phi$ the family of all mappings $\phi: \omega \longrightarrow[\omega]^{<\omega}$ such that, for each $n \in \omega, n \in \phi(n)$ and $\{m: n \in \phi(m)\}$ is finite. We consider the family $\mathcal{F}$ of all subsets of $\omega \times \omega$ of the form

$$
\{(n, k): k \in \phi(n), n \in \omega\}, \phi \in \Phi
$$

and note that $\mathcal{F}$ is a coarse structure on $\omega$, see [11, Example 1.4.6]. The universal property of $(\omega, \mathcal{F})$ : if $\mathcal{E}$ is a coarse structure on $\omega$ such that every bounded subset in $(\omega, \mathcal{E})$ is finite then each injective mapping $f:(\omega, \mathcal{E}) \longrightarrow(\omega, \mathcal{F})$ is macro-uniform.

Theorem 1. $\operatorname{Asdim}(\omega, \mathcal{F})=1$.

Proof. We take an arbitrary $F \in \mathcal{F}$ such that $F=F^{-1},(n, n+1) \in F, n \in \omega$. We put $P_{0}=F[0], P_{n+1}=F\left[P_{n}\right] \backslash\left(P_{0} \cup \cdots \cup P_{n}\right)$ and note that $\cup_{n<\omega} P_{n}=\omega$. We denote $\mathcal{A}_{0}=\left\{P_{2 n}: n<\omega\right\}, \mathcal{A}_{1}=\left\{P_{2 n+1}: n<\omega\right\}$ and observe that $\mathcal{A}_{0}, \mathcal{A}_{1}$ are $F$-disjoint.

We define a mapping $\phi \in \Phi$ by $m \in \phi(n)$ if and only if $m \in P_{n}$. We put $H=\{(n, m): m \in \phi(n), n<\omega\}$ and note that the family $\left\{P_{n}: n<\omega\right\}$ is $H$ bounded so $\operatorname{asdim}(\omega, \mathcal{F}) \leq 1$. Since $(n, n+1) \in F, \omega$ can not be partitioned into $F$-disjoint uniformly bounded subsets, $\operatorname{so} \operatorname{asdim}(\omega, \mathcal{F}) \neq 0$ and $\operatorname{asdim}(\omega, \mathcal{F})=1$. 
Theorem 2. Let $G$ be a subgroup of a topological group $H$ such that there exists an injective sequence $\left(a_{n}\right)_{n<\omega}$ in $G$ converging to $h \in H \backslash G, A=\left\{a_{n}: n<\omega\right\}$. Then the subspace $A$ of $(G, \mathcal{C})$ is asymorphic to $(\omega, \mathcal{F})$.

Proof. We show that the mapping $f:(\omega, \mathcal{F}) \longrightarrow A, f(n)=a_{n}$ is an asymorphism. Since $h \notin G$, each bounded subset of $A$ is finite. By the universal property of $(\omega, \mathcal{F}), f^{-1}$ is macro-uniform.

To prove that $f$ is macro-uniform, we take an arbitrary mapping $\phi: A \longrightarrow[A]^{<\omega}$ such that $a_{n} \in \phi\left(a_{n}\right)$ and $\left\{a_{m}: a_{n} \in \phi\left(a_{m}\right)\right\}$ is finite. We put $K=\cup_{n<\omega} \phi\left(a_{n}\right) a_{n}^{-1}$. By the choice of $\phi$, every injective sequence in $K$ converges to the identity of $G$. Hence, $K$ is compact and $\phi\left(a_{n}\right) \subseteq K a_{n}$.

Theorem 3. For every non-discrete countable metrizable abelian topological group $G$, $\operatorname{asdim}(G, \mathcal{C})=\infty$.

Proof. We fix a natural number $m$ and prove that $\operatorname{asdim}(G, \mathcal{C}) \geq m$. Since the completion $H$ of $G$ is uncountable, we can choose $h_{1}, \ldots, h_{m} \in H \backslash G$ such that

(1) $i_{1} h_{1}+\cdots+i_{m} h_{m} \in G,\left(i_{1}, \ldots, i_{m}\right) \in\{-1,0,1\}^{m}$ implies $i_{1}=\cdots=i_{m}=0$.

Then we choose injective sequences $\left(a_{1 n}\right)_{n<\omega}, \ldots,\left(a_{m n}\right)_{n<\omega}$ converging to $h_{1}, \ldots, h_{m}$ such that

(2) $a_{1 i_{1}}+\cdots+a_{m i_{m}}=a_{1 j_{1}}+\cdots+a_{m j_{m}}$ implies $i_{1}=j_{1}, \ldots, i_{m}=j_{m}$.

By (2), for $A=A_{1}+\cdots+A_{m}, A_{i}=\left\{a_{i n}: n<\omega\right\}$, the mapping $f: A \longrightarrow$ $(\omega, \mathcal{F})^{m}, f\left(a_{1 i_{1}}+\cdots+a_{m i_{m}}=\left(i_{1}, \ldots, i_{m}\right)\right.$ is bijection. By Theorem 1 and [1], $\operatorname{asdim}(\omega, \mathcal{F})^{m} \leq m$, so it suffices to prove that $f$ is an asymorphism. Applying Theorem 2 , we see that $f^{-1}$ is macro-uniform.

To prove that $f$ is macro-uniform, we take an arbitrary compact $K$ in $G, 0 \in K$ and show that there exist $\phi_{1}: A_{1} \longrightarrow A_{1}^{<\omega}, \ldots, \phi_{m}: A_{m} \longrightarrow A_{m}^{<\omega}$ such that

(3) $a \in \phi_{i}(a)$, and $\left\{b \in A_{i}: a \in \phi_{i}(b)\right\}$ is finite, $a \in A_{i}, i \in\{1, \ldots, m\}$;

(4) $A \cap\left(K+a_{1 i_{1}}+\cdots+a_{m i_{m}}\right) \subseteq \phi_{1}\left(a_{1 i_{1}}\right)+\cdots+\phi_{m}\left(a_{m i_{m}}\right)$.

We fix $k \in\{1, \ldots, m\}, j \in \omega$ and denote by $\phi_{k}\left(a_{k j}\right)$ the set of all $a_{k s} \in A_{k}$ such that $\left(A_{1}+\cdots+A_{k-1}+a_{k j}+A_{k+1}+\cdots+A_{m}\right) \cap\left(K+A_{1}+\cdots+A_{k-1}+a_{k s}+\right.$ $\left.A_{k+1}+\cdots+A_{m}\right) \neq \emptyset$. 
Applying (1), we conclude that $\phi_{k}\left(a_{k j}\right)$ is finite and $\phi_{1}, \ldots, \phi_{m}$ satisfy $(3),(4)$.

\section{REFERENCES}

[1] I. Banakh, T. Banakh, On the asymptotic dimension of coarse spaces, arXiv: 2001.04300.

[2] D. Dikranjan, N. Zava, Some categorical aspects of coarse spaces and balleans, Topology Appl. 225(2017), 164-194.

[3] D. Dikranjan, N. Zava, The impact of Pontryagin and Bohr functors on large-scale properties of LCA-groups, Topology Appl. (to appear).

[4] P. de la Harpe, Topics in Geometric Group Theory, Chicago Lectures in Math, University of Chicago Press, Chicago, 2000.

[5] S. Hernandes, I. V. Protasov, Balleans of topological groups, J. Math. Sciences 178 (2011), no. $1,65-74$.

[6] A. Nicas, D. Rosenthal, On the asymptotic dimension of the dual group of a locally compact abelian group, Topology Appl. 160 (2013), 682-684.

[7] I.V. Protasov, Sequential coarse structures of topological groups, Math. Stud. 51 (2019), $12-18$.

[8] I. Protasov, T. Banakh, Ball Structures and Colorings of Groups and Graphs, Mat. Stud. Monogr. Ser 11, VNTL, Lviv, 2003.

[9] I.V. Protasov, O.I. Protasova, Sketch of group balleans, Math. Stud. 22 (2004), 10-20.

[10] I. Protasov, S. Slobodianiuk, On asymorphisms of groups, J. Group Theory 20 (2017), 393-399.

[11] I. Protasov, M. Zarichnyi, General Asymptopogy, Math. Stud. Monogr. Vol. 12, VNTL, Lviv, 2007.

[12] J. Roe, Lectures on Coarse Geometry, Univ. Lecture Ser., vol. 31, American Mathematical Society, Providence RI, 2003.

\section{CONTACT INFORMATION}

I. Protasov:

Faculty of Computer Science and Cybernetics

Kyiv University

Academic Glushkov pr. 4d

03680 Kyiv, Ukraine

i.v.protasov@gmail.com 